\section{El Programa Ambiente y Sociedad de la Universidad Nacional del Litoral: 15 años re-construyendo las relaciones naturaleza-cultura}

\author{
Enrique Raúl Mihura \\ enriqueraulmihura@hotmail.com.ar \\ (iD) orcid.org/0000-0001-7430-1023 \\ Alba Imhof \\ aimhof@fhuc.unl.edu.ar \\ (iD) orcid.org/0000-0003-0317-9299
}

\author{
Patricia Mines \\ pmines@fadu.unl.edu.ar \\ (iD) orcid.org/0000-0001-5220-4185
}

Programa Ambiente y Sociedad de la

Secretaría de Extensión Social y Cultural,

Universidad Nacional del Litoral, Argentina.
Ambiente y extensión universitaria /

Desafíos de gestión

다(1)(2)(2)
RECEPCIÓN: 29/04/21

ACEPTACIÓN FINAL: 30/06/21

\section{Resumen}

El ambiente es un concepto transdisciplinar que atraviesa la vida de las comunidades y que la Universidad Nacional del Litoral ha institucionalizado a través del Programa Ambiente y Sociedad. Este artículo presenta algunas consideraciones sobre el concepto de ambiente que hace suyas el Programa de la Secretaría de Extensión Social y Cultural y orientan la definición de los objetivos, líneas de acción y las principales estrategias de articulación en extensión, docencia e investigación que lleva adelante. Se desarrolla el caso del laboratorio a cielo abierto Microrregión Insular Santa Fe, San José del Rincón y Arroyo Leyes. Se concluye con una reflexión del camino recorrido destacando los desafíos de gestión, limitaciones y logros en la reconstrucción continua de las relaciones del ambiente y la sociedad.

Palabras clave: programa de extensión; ambiente y sociedad; desarrollo sustentable; Universidad Nacional del Litoral; extensión universitaria.
The Environment and Society Program of the Universidad Nacional del Litoral: 15 years re-building nature-culture relationships

\begin{abstract}
The Environment is a transdisciplinary concept that runs through the life of the communities and that the Universidad Nacional del Litoral has institutionalized through the Environment and Society Program. This article presents some considerations on the concept of Environment endorsed by the Program of the Secretariat of Social and Cultural Extension and guides the definition of the objectives, lines of action and the main strategies of articulation in extension, teaching and research that it carries out. The case of the open-air laboratory Micro Región Insular Santa Fe, San José del Rincón and Arroyo Leyes is developed. It concludes with a reflection of the path traveled, highlighting the management challenges, limitations and achievements in the continuous reconstruction of the relations of the Environment and Society. It concludes with some thoughts on the experiences had, highlighting the management challenges, limitations, and achievements in the continuous reconstruction of the relationship between environment and society.

Keywords: extension program; environment and society; sustainable development; Universidad Nacional del Litoral; university extension.
\end{abstract}

O Programa Ambiente e Sociedade da Universidade Nacional del Litoral: 15 anos reconstruindo as relações natureza-cultura

\section{Resumo}

O ambiente é um conceito transdisciplinar que permeia a vida das comunidades e que a Universidade Nacional del Litoral institucionalizou por meio do Programa Ambiente e Sociedade. Este artigo apresenta algumas considerações sobre o conceito de ambiente endossado pelo Programa da Secretaria de Extensão Social e Cultural e orientam a definição dos objetivos, linhas de ação e as principais estratégias de articulação em extensão, ensino e pesquisa que realiza. Desenvolvese o caso do laboratório a céu aberto Micro Región Insular Santa Fe, San José del Rincón e Arroyo Leyes. Conclui-se com uma reflexão sobre o caminho percorrido, destacando os desafios, limitações e conquistas da gestão na reconstrução contínua das relações do ambiente e a sociedade.

Palavras-chave: programa de extensão; desenvolvimento sustentável; Universidade Nacional del Litoral; extensão universitária; ambiente e sociedade.

Para citación de este artículo: Mihura, E,; Imhof, A. y Mines, P. (2021). El Programa Ambiente y Sociedad de la Universidad Nacional del Litoral: 15 años re-construyendo las relaciones naturaleza-cultura. +E: Revista de Extensión Universitaria, 11(14), e0015. doi: 10.14409/extension.2021.14. Ene-Jun.e0015 


\section{Introducción}

El Programa Ambiente y Sociedad (PAS) se desarrolla desde 2006 en el ámbito de la Secretaría de Extensión de la Universidad Nacional del Litoral (UNL), hoy Secretaría de Extensión Social y Cultural (SESyC), e integra visiones, disciplinas y problemáticas del patrimonio natural y cultural del área de la Universidad. El presente artículo se propone reflexionar sobre el recorrido de este dispositivo de gestión institucional a la luz de los desafíos epistemológicos y metodológicos que vienen planteando los conflictos ambientales en los últimos años. En este marco se repasan las principales consideraciones sobre: el concepto de ambiente, el desarrollo sustentable como idea fuerza del paradigma ambiental, y se aporta un esquema sobre las miradas de los principales autores que, desde la perspectiva del PAS, constituyen el fundamento de sus acciones actuales. Se plantean la visión, los objetivos y estrategias actuales del programa y se destacan los conceptos de laboratorio a cielo abierto e investigación interactiva que se presentan al lector como las innovaciones que integran las funciones universitarias de investigación, extensión y docencia. Sobre la investigación interactiva se ofrece un cuadro de los procesos involucrados. Se enumeran los espacios de docencia y algunos proyectos de extensión vinculados. El caso del laboratorio a cielo abierto: Microrregión Insular constituye uno de los escenarios más ricos para la integración deseada, en donde se repasan logros y dificultades.

\section{Algunas consideraciones sobre ambiente}

Estamos atravesando una nueva era, el Antropoceno (Crutzen \& Stoermer, 2000), un concepto que aún se encuentra en discusión pero que se basa en la idea de que el hombre ha realizado en el último período de la historia modificaciones de tal magnitud en los procesos naturales que puede ser considerado una fuerza geológica. Si no se producen cambios importantes, la humanidad en continuo crecimiento y acercándose a los 10000 millones de habitantes, se llegará a un punto de no retorno.

Las soluciones se encuentran en la modificación de las formas en que nos relacionamos con lo que nos rodea, la relación sociedad-ambiente. El ambiente como objeto de estudio e intervención, y como la mayoría de los fenómenos complejos, admite distintas definiciones, contextuales y operativas. Puede ser interpretado como un sistema global de elementos heterogéneos e interactivos de carácter físico, biótico, social y cultural que configura el espacio donde ocurre todo (Bru, 1997); ambiente como unidad de desarrollo, como lugar y tiempo en donde sucede la vida (Mihura, 2020); sistema complejo, con componentes y relaciones, es decir, se puede leer el ambiente —ni más, ni menos-que como la interacción permanente entre los factores físicos, bióticos, abióticos, humanos, históricos, culturales (Pesci et. al., 2007). Y estas interrelaciones pueden ser positivas o negativas, a diversas escalas y en evolución conjunta constante (Folch y Bru, 2017).

En la actualidad, el proceso determinante de lo que ocurre o podría ocurrir en el sistema ambiental planetario es el de la globalización, que ha sido definida como un fenómeno inevitable en la historia humana que ha acercado el mundo a través del intercambio de bienes y productos, información, conocimientos y cultura. En las últimas décadas, esta integración mundial ha cobrado velocidad de forma espectacular debido a los avances sin precedentes en la tecnología, las comunicaciones, la ciencia, el transporte y la industria. La Organización 
de las Naciones Unidas (ONU) reconocen que la tarea fundamental es que "la globalización se convierta en una fuerza positiva para todos los habitantes del mundo ya que, si bien la misma ofrece grandes posibilidades, en la actualidad sus beneficios y costos de distribuyen de manera muy desigual" (ONU, 2010, p. 7).

Frente a estos procesos globales, los esfuerzos locales son los que pueden transformar la realidad. La organización, reorganización y desarrollo de bloques regionales, el fortalecimiento de los Estados nacionales, las microrregiones y las comunidades locales organizadas se constituyen en los sistemas de gestión y planificación del desarrollo sustentable que habiliten la puesta en marcha e implementación de los procesos de reconfiguración naturaleza-cultura.

El desarrollo sustentable es la idea fuerza que orienta el paradigma ambiental. La traducción del inglés del término sustainable abrió un debate en el cual se identifican dos posiciones: el uso indistinto de las palabras sostenible y sustentable (Gallopín, 2003) o la oportunidad de indagar en el significado de cada uno de los términos con relación a los estilos de desarrollo. En este segundo grupo se encuentran Ruben Pesci y FLACAM, Red de Cátedras Unesco para el Desarrollo Sustentable. FLACAM asimila el significado del término sostenible a un proceso de transformación de un ambiente que debe ser sostenido para mantener sus condiciones actuales en el tiempo (Pesci, 2010). Hacer sostenible el desarrollo significa, en este contexto, mantener las condiciones actuales de ese desarrollo, mantener las condiciones con sus inequidades y desigualdades. La sustentabilidad como estilo de desarrollo refiere, en cambio, al sistema que ha adquirido las capacidades de sostenerse y, además, ha logrado tener las capacidades superadoras para alcanzar un mayor desarrollo y mejor calidad de vida. El concepto de resiliencia se vincula aquí a la adaptabilidad de un sistema como propuesta de mejora en la calidad de vida, es decir, de proyecto alternativo.

La clave del concepto de desarrollo sustentable es que busca equilibrar conservación y desarrollo a través de conciliar sus diversas dimensiones: los pilares ecológico, social y económico a los que Gallopín (2006) agrega la dimensión institucional (o de gestión). Molina Neira (2018) recopila lo que afirman diversos autores respecto de la necesidad de incorporar la cultura como nuevo pilar, considerada como la manifestación y práctica de la cultura tradicional y nueva, como resultado de un constante proceso creativo y de libre expresión individual y colectiva que enlaza pasado y presente en el espacio físico artificial y natural y genera una dinámica que caracteriza la solución de problemas, la forma de vida, el arte, el conocimiento, el patrimonio tangible e intangible y, especialmente, la identidad, lo cual en conjunto conforma un legado que se espera llegue a futuro.

Revalorizar el capital cultural (Bordieu, 2011) y los recursos patrimoniales (Sabaté Bel, 2004) puede suponer un modelo más viable en cuanto a lo económico, ambientalmente sostenible atento a la identidad de cada territorio y socialmente más justo.

Estas conceptualizaciones y definiciones quedaron sistematizadas en los Objetivos de Desarrollo Sostenible (ODS) que fueron aprobados en la Cumbre Mundial sobre el Desarrollo Sostenible realizada en la ONU en Nueva York en septiembre de 2015, a través de la iniciativa internacional "Transformar nuestro mundo: la Agenda 2030 para el Desarrollo Sostenible".

"Los Objetivos de Desarrollo Sostenible son el plan maestro para conseguir un futuro sostenible para todos. Se interrelacionan entre sí e incorporan los desafíos globales a los que nos enfrentamos día a día, como la pobreza, la desigualdad, el clima, la degradación 
ambiental, la prosperidad, la paz y la justicia. Para no dejar a nadie atrás, es importante que logremos cumplir con cada uno de estos objetivos para 2030". (ONU, 2018)

Aunque los ODS no son legalmente vinculantes, han tenido una buena acogida y un relativo impacto sobre las estrategias y acciones de los gobiernos, las empresas y las organizaciones de la sociedad civil.

\section{El contexto institucional de la UNL}

La UNL nace al calor de la renovación social, cultural y política de comienzos del siglo XX que desencadena la Reforma Universitaria de 1918.

"La Universidad Nacional del Litoral constituye una república universitaria que, comprometida con los postulados que le dieron origen, lucha por la generación y distribución del conocimiento como un bien público y social, asumiendo el desafío de formar mujeres y hombres libres que, respetuosos de los derechos inviolables e inalienables de la persona humana y el desarrollo sustentable así como la defensa de los valores democráticos, trabajen por una Argentina inclusiva, solidaria, con mayor libertad, igualdad, equidad y justicia e integrada a Latinoamérica y al mundo". (UNL, 2013).

A través de la articulación de las funciones sustantivas de enseñanza, investigación y extensión, y de las políticas institucionales en las cuales se traduce especialmente esta concepción democrática que pone en juego el diálogo entre conocimientos científicos con saberes y conocimientos del medio, busca aportar soluciones a situaciones problemáticas con actitud crítica, creativa y transformadora. La extensión, por medio de sus prácticas, ha sido la puerta de entrada al compromiso social, lo que se evidencia en acciones, territorios y actores (Menéndez y Tarabella, 2017).

"La extensión universitaria representa el compromiso de la Universidad Nacional del Litoral para con el medio social, productivo y cultural del cual se nutre y forma parte, y se materializa a través de políticas e instrumentos de gestión, así como de una profunda integración con la enseñanza y la investigación y desarrollo, a los fines de posibilitar la generación, transmisión, transferencia, intercambio, circulación y apropiación social de conocimientos. (Capítulo 3, artículo 82, Estatuto UNL, 2013)

Dentro de la estructura de la SESyC, los programas de extensión forman parte de la política de extensión que se lleva adelante en el marco del Sistema Integrado de Programas, Proyectos y Prácticas (SIPPPE) ${ }^{1}$. El objetivo principal de la integración de Programas, proyectos y prácticas es realizar aportes significativos al fortalecimiento de la democracia, a la promoción del desarrollo territorial, a la inclusión social; a la mejora en la calidad de vida y al bienestar de los ciudadanos, en el marco de las políticas públicas. Los Programas constituyen dispositivos políticos y de gestión que abordan específicamente determinadas 
problemáticas consideradas significativas para la Universidad y forman parte de las preocupaciones más importante de la agenda pública.

El Programa Ambiente y Sociedad (PAS), es uno de estos dispositivos políticos y de gestión que se inicia en el año 2006, con el objetivo específico de contribuir a la resolución de problemas ambientales integrando a todos los actores del medio social, productivo y cultural - del cual la universidad forma parte — promoviendo la producción y distribución del conocimiento como bien público como fuerza transformadora de la sociedad. Desde sus comienzos ha intentado trascender conceptos sectoriales siendo ámbito de reflexión crítica. Los debates y posicionamientos sostenidos durante estos años fueron claves para definir la visión, los objetivos y los mecanismos de funcionamiento de este dispositivo institucional.

\section{EI PAS}

Este programa tiene por misión facilitar el diálogo de saberes, la producción de conocimientos y el desarrollo de tecnologías de gestión de manera concertada para favorecer la organización, gestión y planificación de procesos de desarrollo socioeconómico con restricciones (desarrollo sustentable). Intenta ser un ensayo/demostración de factibilidad de funcionamiento universitario transdisciplinario cuya premisa central es contribuir a mejorar la calidad de vida desde una perspectiva/enfoque biocéntrica/o.

Su estructura de gestión está conformada por una dirección con sede en la Facultad de Ingeniería y Ciencias Hídricas (FICH) de la UNL y dos coordinaciones que abordan de modo integral la problemática ambiental desde la perspectiva del patrimonio, complementaria y operativamente dividida en patrimonio natural y cultural.

EI PAS ha logrado desde su creación un desarrollo innovador en la integración de aspectos culturales y naturales en un tiempo de renovados desafíos. Ha avanzado sobre conceptos tradicionalmente sectoriales de sociedad, de naturaleza, de patrimonio, de gobernanza, logrando una articulación entre saberes y disciplinas. Hace suyas las premisas y deseabilidades que comunican los ODS. Plantea una visión ampliada e integral, que debate y pone a prueba los conceptos mismos de ambiente y desarrollo sustentable. A través de estrategias de articulación, diálogo de saberes y gestión interactiva para el desarrollo sustentable como idea fuerza del paradigma ambiental, se avanza sistemáticamente en propuestas sobre universidades necesarias para el cambio asociadas a un modelo transdisciplinario y la visión de sistemas y la sustentabilidad. ${ }^{2}$

\footnotetext{
2) Un modelo de funcionamiento transdisciplinario deseado y posible fue expuesto por primera vez en el año 1993 en el Consejo Superior de la UNL y luego publicado en Proyectar la sustentabilidad (Pesci, et al., 2007) constituye un antecedente del convenio FLACAM/UNL A través del Programa Ambiente y Sociedad se otorga provisionalmente a la UNL la condición de Miembro Adherente de la Red FLACAM, según lo expresado en el Convenio Marco de Colaboración entre la UNL y el Foro Latinoamericano de Ciencias Ambientales (Cátedra Unesco para el Desarrollo Sustentable) de 2010, con el objeto de colaborar en materia de diseño y ejecución de proyectos, formación profesional, transferencia del saber y difusión de una conciencia ambiental.
} 
Figura 1. PAS y desarrollo sustentable

\begin{tabular}{|c|c|c|c|c|c|c|c|c|}
\hline \multicolumn{9}{|c|}{ Desarrollo sustentable: Idea fuerza que orienta el paradigma ambiental } \\
\hline \multirow[t]{7}{*}{$\begin{array}{l}\text { Se puede } \\
\text { comprender } \\
\text { como }\end{array}$} & \multicolumn{8}{|c|}{$\begin{array}{l}\text { Un acto de responsabilidad y de solidaridad inter-generacional relacionado con la sobrevivencia } \\
\text { de nuestra especie. Es el desarrollo que satisface las necesidades de la generación presente sin comprometer } \\
\text { la capacidad de las generaciones futuras para satisfacer sus propias necesidades (CAMD UN, 1987) }\end{array}$} \\
\hline & \multicolumn{8}{|c|}{$\begin{array}{l}\text { Un medio para mejorar la calidad de vida, entendida cómo el grado de satisfacción de necesidades humanas } \\
\text { fundamentales (Max Neef, 1993), en un marco de conservación del patrimonio natural y cultural. }\end{array}$} \\
\hline & \multicolumn{8}{|c|}{$\begin{array}{l}\text { Un camino para la construcción de una sociedad sustentable: buen vivir, vivir bien, flujos cíclicos } \\
\text { (Pesci R., et. al., 2007), combinaciones. }\end{array}$} \\
\hline & \multicolumn{8}{|c|}{ Un proceso de crecimiento económico con equidad y sustentabilidad ambiental (Axel Dourognanni, CEPAL). } \\
\hline & \multicolumn{8}{|c|}{$\begin{array}{l}\text { Un proceso de cambio direccional, mediante el cual un sistema mejora de manera sustentable a través } \\
\text { del tiempo. Este "proceso de cambio resulta asemejable al concepto de despliegue cualitativo de potencialidades } \\
\text { de complejidad creciente" (Gallopín, 2003:21) }\end{array}$} \\
\hline & \multicolumn{8}{|c|}{$\begin{array}{l}\text { Un desarrollo socio-económico con restricciones. El cual encierra dos conceptos fundamentales (CAMD UN, } \\
\text { 1987): el de necesidades (en particular las necesidades esenciales de los pobres) y la idea de limitaciones } \\
\text { impuestas por el estado de la tecnología y la organización social. Requiere para su implementación: }\end{array}$} \\
\hline & $\begin{array}{l}\text { Un modelo } \\
\text { internacional } \\
\text { que promueva } \\
\text { constantemen- } \\
\text { te modelos } \\
\text { duraderos } \\
\text { de comercio } \\
\text { y finanzas. }\end{array}$ & $\begin{array}{l}\text { Un siste- } \\
\text { ma político } \\
\text { democrático } \\
\text { que asegure a } \\
\text { sus ciudadanos } \\
\text { una participa- } \\
\text { ción efectiva } \\
\text { en la toma de } \\
\text { decisiones. }\end{array}$ & $\begin{array}{l}\text { Un sistema de } \\
\text { organización } \\
\text { social cuya } \\
\text { lógica de } \\
\text { funcionamiento } \\
\text { (racionalidad) } \\
\text { (Leff, E., } \\
\text { 2013) sea } \\
\text { alternativa a las } \\
\text { provistas por el } \\
\text { capitalismo y } \\
\text { la planificación } \\
\text { descentra- } \\
\text { lizada. }\end{array}$ & $\begin{array}{l}\text { Un sistema } \\
\text { administrativo } \\
\text { flexible y capaz } \\
\text { de corregirse } \\
\text { de manera } \\
\text { autónoma. }\end{array}$ & $\begin{array}{l}\text { Un sistema } \\
\text { económico } \\
\text { capaz de crear } \\
\text { excedentes y } \\
\text { conocimien- } \\
\text { to técnico } \\
\text { sobre una base } \\
\text { autónoma y } \\
\text { constante. }\end{array}$ & $\begin{array}{l}\text { Un sistema de produc- } \\
\text { ción de micro y pequeñas } \\
\text { empresas, generadoras } \\
\text { de empleo y facilitadoras } \\
\text { de distribución de las } \\
\text { ganancias. Donde las } \\
\text { rentabilidades necesarias } \\
\text { para la sostenibilidad de las } \\
\text { empresas sean compati- } \\
\text { bles con velocidades de } \\
\text { aprovechamiento de los } \\
\text { recursos, que se encuentren } \\
\text { por debajo de las tasas de } \\
\text { reposición de los mismos, } \\
\text { con el propósito de que } \\
\text { cumpla con el imperativo } \\
\text { de cuidar el ambiente. }\end{array}$ & $\begin{array}{l}\text { Un sistema } \\
\text { social que evite } \\
\text { las tensiones } \\
\text { provocadas por } \\
\text { un desarrollo } \\
\text { desequilibrado. }\end{array}$ & $\begin{array}{l}\text { Un sistema tec- } \\
\text { nológico capaz } \\
\text { de investigar } \\
\text { constante- } \\
\text { mente nuevas } \\
\text { soluciones }\end{array}$ \\
\hline
\end{tabular}

Fuente: PAS.

El objetivo general del PAS es contribuir a la resolución de problemas ambientales que se plantean como desafíos en la sociedad actual y promover la producción y transferencia de conocimientos, la búsqueda de formas innovadoras de imaginar la protección y construcción social-territorial a través de estrategias de articulación, diálogo de saberes y gestión interactiva para el desarrollo sustentable.

Entre sus objetivos específicos se destacan:

- Reflexionar sobre el concepto de desarrollo sustentable, la protección de áreas naturales, los estilos de desarrollos posibles y socialmente acordados.

- Promover el diálogo de saberes y la reflexión crítica desde el enfoque biocéntrico y de sistemas complejos, articulando docentes, investigadores y extensionistas entre sí y con diferentes actores sociales.

- Propiciar la formación de estudiantes y docentes en el conocimiento y manejo de los sistemas ambientales y gestionar a través de actividades formales y no formales la incorporación de la dimensión ambiental a todos los niveles de educación y de la comunidad. - Avanzar en la categorización y puesta en valor de las áreas protegidas existentes e impulsar la implementación de nuevas modalidades de protección y desarrollo comunitario.

- Promover el enfoque "Proyectar ambientes" como modelo de intervención puesto a prueba en experiencias de abordaje de sistemas a diferente escala. 


\section{Líneas de trabajo}

Sus líneas de trabajo han ido cambiando de acuerdo con la impronta de los diversos directores que lo tuvieron a su cargo. ${ }^{3}$ Áreas naturales, flora y fauna como temas centrales en su origen se diversificaron más adelante con las problemáticas en torno a los agroquímicos y la contaminación. Actualmente, el proceso de gestión se encuentra organizado en cuatro líneas principales de trabajo:

- Educación ambiental y Educación para el Desarrollo Sustentable.

- Gestión de Áreas Protegidas: políticas públicas de conservación y cuidado. Relación universidad y movimientos sociales para la defensa de los espacios naturales.

- Gestión y Planificación del Desarrollo Sustentable: instrumentos de gobernanza, gestión de la tensión conservación-desarrollo, iniciativas de colaboración entre la Universidad, gobiernos y actores de la sociedad civil. Estrategias para la gestión participativa de bienes comunes (patrimonio natural y cultural).

- Gestión Integral de Riesgo y políticas ambientales: participación en la construcción de políticas públicas, monitoreo y evaluación.

EI PAS es un dispositivo institucional de articulación con los actores del desarrollo del ambiente regional en el que la Universidad se encuentra inserta. Conforma redes colaborativas internas en la UNL, equipos interdisciplinarios y redes de cooperación interinstitucionales.

A través de la planificación y coordinación de diversas actividades (congresos, seminarios, jornadas, talleres, conversatorios), proyectos de investigación y extensión, pasantías, adscripciones y cientibecas, prácticas de educación experiencial, dictado de asignaturas, etc., orientados de temas prioritarios acordados entre la comunidad y la Universidad, ha ido abordando las líneas de trabajo en un cruce sinérgico de propuestas. Educación inclusiva, equitativa y de calidad (educación para el desarrollo y estilos de vida sostenibles, promoción de la democratización de la cultura y valoración de la diversidad); Energías asequibles y no contaminantes (utilización de tecnologías alternativas); Ciudades y comunidades sostenibles (asentamientos seguros, inclusivos y resilientes, acceso a zonas verdes y espacio público, participación ciudadana, protección y preservación del patrimonio cultural y natural, movilidad urbana); Producción y consumo responsable (hábitos de consumo y prácticas asociadas a modos de vida sustentable, emprendimientos productivos locales, turismo sustentable, y comunitario, piscicultura, gestión integrada de residuos, reciclaje de papel y plástico promoción de instalación de jardines familiares); Ambientes y ecosistemas sostenibles (áreas protegidas, biodiversidad local, saneamiento ambiental, calidad de agua, manejo de plaguicidas, conservación de bosques nativos, puesta en valor de especies locales) y Cultura, patrimonio y creatividad (desarrollo de la diversidad cultural de las comunidades y grupos sociales, desde una concepción holística e integrada de forma operativa a los procesos de desarrollo sostenible, patrimonio (tangible e intangible) como recurso para el desarrollo sostenible en ciudades y regiones, dimensión simbólica de las prácticas sociales)

Este programa se caracteriza por la continua actuación en ámbitos colaborativos: con gobiernos provinciales, municipios, comunas, organizaciones de la sociedad civil y parti- 
culares interesados, en la resolución de problemáticas actuales. Es así que ha participado activamente en los procesos de creación del Parque Natural Islas Santa Fe, Reserva Urbana Oeste de la ciudad de Santa Fe, Microrregiones Insulares de Santa Fe, San José del Rincón y Arroyo Leyes y de la Cuenca del arroyo Los Padres. Participa en los Comités de manejo de las reservas de la Ciudad Universitaria en Santa Fe y la Reserva Natural de la Escuela de Agricultura, Ganadería y Granja Med. Vet. Martín Rodolfo de la Peña, en Esperanza, ambas de propiedad de la UNL. En el año 2019 se presentó un proyecto al Ministerio de Ambiente de la provincia de Santa Fe con una propuesta de creación de una Reserva Hídrica que constituiría una estrategia a largo plazo para la conservación de la biodiversidad natural y cultural en una zona de islas del departamento La Capital. Desde 2010 se impulsa la iniciativa de Reserva de Biosfera en Ambiente Urbano para la Región Metropolitana Santa Fe-Paraná.

\section{Estrategias del PAS}

A lo largo de todos estos años, el PAS ha ido adecuando sus estrategias y proponiendo metodologías para la implementación de sus experiencias y nuevos desarrollos inherentes a la puesta en marcha de laboratorios a cielo abierto y procesos de investigación interactiva.

\section{Laboratorios a cielo abierto y procesos de investigación interactiva}

Desde el punto de vista de la extensión, cualquier práctica que la universidad defina con la comunidad pone en juego el aspecto relacional (Menéndez, 2013), y a partir de este diálogo se construyen escenarios conjuntamente con organizaciones sociales y organismos públicos, se establecen redes de relaciones en las que se plantean premisas, se enumeran conflictos y potencialidades, se reconocen beneficiarios y se identifican temas generadores de proyectos transformadores. Hoy le cabe a la extensión el rol de traccionar cambios e innovaciones, tanto hacia afuera como hacia adentro de la misma institución universitaria, que trascienden los espacios de extensión, investigación y docencia como compartimentos estanco. En particular, las experiencias ejecutadas y en ejecución parten de considerar que los ámbitos convencionales donde se lleva a cabo la investigación sobre procesos de desarrollo sustentable deben ser replanteados/readecuados.

El planteo de los laboratorios a cielo abierto surge del PAS para desarrollar y perfeccionar experiencias de desarrollo sustentable en sistemas ambientales con territorios y actores concretos. Se proponen como escenarios reales en los cuales el sistema de muestreo y análisis debe incluir todos los saberes disponibles; los enfoques y metodologías de investigación tienen que ser interactivos con el medio, y las verificaciones relacionadas con los desarrollos de nuevos sistemas y/o innovaciones en sistemas preexistentes deben producirse sobre la base de resultados alcanzados en términos de mejorar la calidad de vida.

El proceso de investigación interactiva diseñado y utilizado por el equipo del PAS en estos escenarios para la producción de nuevos conocimientos integra las tres funciones sustantivas del quehacer universitario actual (enseñanza, investigación y extensión), los servicios a terceros y la gestión institucional, mediante tres procesos alrededor de los cuales el equipo organiza su funcionamiento colectivo, a saber: 
Figura 2. Procesos de la gestión interactiva

\begin{tabular}{|c|c|c|c|}
\hline $\begin{array}{l}\text { Diálogo } \\
\text { de saberes } \\
\text { y gestión }\end{array}$ & \multicolumn{3}{|c|}{$\begin{array}{l}\text { Es la realización de situaciones de trabajo (conversaciones, reuniones, talleres, seminarios, } \\
\text { cursos) con los autores del desarrollo del sistema ambiental definido como unidad de } \\
\text { actuación que debe ser oportunamente proyectado/diseñado. }\end{array}$} \\
\hline \multirow[t]{5}{*}{$\begin{array}{l}\text { Producción } \\
\text { de contenidos }\end{array}$} & \multirow{4}{*}{$\begin{array}{l}\text { Proyectar ambientes } \\
\text { (servicios de asisten- } \\
\text { cia técnica/una forma } \\
\text { de proyectar): generar } \\
\text { información sobre el lugar, } \\
\text { elaborar programas, pro- } \\
\text { yectos, presupuestos, etc., } \\
\text { para resolver problemas, } \\
\text { lo que vuelve sostenible } \\
\text { la gestión interactiva y } \\
\text { posibilita a través de ella } \\
\text { el enriquecimiento, mejor } \\
\text { desarrollo y mayor consis- } \\
\text { tencia de los programas/ } \\
\text { proyectos formulados. }\end{array}$} & \multirow{2}{*}{$\begin{array}{l}\text { Comprender } \\
\text { el ambiente } \\
\text { seleccionado es } \\
\text { "desarmarlo", } \\
\text { progresivamente, } \\
\text { según dos niveles }\end{array}$} & $\begin{array}{l}\text { Primer nivel de comprensión (diagnóstico): } \\
\text { descripción, análisis lineal y determinación } \\
\text { de objetivos de política. }\end{array}$ \\
\hline & & & $\begin{array}{l}\text { Segundo nivel de comprensión (diagnóstico): } \\
\text { análisis relacional y determinación del } \\
\text { conflicto más perturbador. }\end{array}$ \\
\hline & & \multirow{2}{*}{$\begin{array}{l}\text { Proyectar/ } \\
\text { implementar } \\
\text { adecuadamente } \\
\text { las transformacio- } \\
\text { nes del ambiente } \\
\text { seleccionado. }\end{array}$} & $\begin{array}{l}\text { Diseñar los ciclos de proyecto a recorrer con } \\
\text { el rumbo y marco que brindan los objetivos } \\
\text { de política determinados. }\end{array}$ \\
\hline & & & $\begin{array}{l}\text { Diseñar/implementar el tema generador - } \\
\text { tema acción- del primer ciclo de proyecto } \\
\text { tomando como insumos las potencialidades } \\
\text { identificadas. Es el comienzo de solución de } \\
\text { las interfases conflictivas identificadas }\end{array}$ \\
\hline & \multicolumn{3}{|c|}{$\begin{array}{l}\text { Elaboración de nuevos conocimientos: análisis y construcción de conocimientos sobre el foco } \\
\text { definido (gestión del desarrollo sustentable), mediante el estudio de situaciones (sincrónico) } \\
\text { y procesos (diacrónico) con indicadores y patrones. }\end{array}$} \\
\hline
\end{tabular}

Fuente: Mihura, 2010.

A partir de 2013, desde la dirección del programa se viene colaborando con el desarrollo de uno de los nodos principales de su red, el equipo de investigación autor y ejecutor de los CAl+D 2011 y 2016 denominados "Procedimientos de Gestión del Desarrollo Sustentable en Áreas Inundables con Gobernabilidad Difusa" y el CAl+D 2020 recientemente aprobado y en ejecución llamado "La Gestión del Desarrollo Sustentable en la Microrregión Insular de Santa Fe, San José del Rincón y Arroyo Leyes (MRI): la gobernanza de la estrategia de desarrollo", con sede en la FICH de la UNL.

El proceso de investigación interactiva surgió por la necesidad de encontrar formas de manejo que eviten la degradación de la gran cantidad de zonas inundables (ambientes de humedales) existentes en la Cuenca del Plata, con algunas características principales como: zonas bajas y periódicamente inundables, aledañas y/o formando parte del valle de inundación de grandes ríos conformadas por espacios continentales, surcados por riachos y arroyos, zonas de islas, selvas en galería; ambientes palustres y lacustres poseedores de alta biodiversidad, prestadores de servicios ambientales; asentamiento de pequeñas localidades, parajes, zonas de recreación de grandes ciudades que reproducen patrones de ocupación y uso del suelo propios de zonas de llanuras altas; periféricas a sistemas urbano-ambientales saturados y expulsores de poblaciones con necesidades básicas insatisfechas y proveniente de clases medias y altas en búsqueda de una mejor calidad de hábitat; objetos del desarrollo turístico a partir de los nuevos enfoques mundiales.

El supuesto principal es que la implementación de "procedimientos de gestión del desarrollo sustentable en áreas inundables con gobernabilidad difusa" implicará la transformación de estos sistemas complejos en "sujetos del desarrollo", con capacidades suficientes para gestionar la implementación de medidas en los sistemas superiores, construir planes, 
programas y proyectos que generen procesos de crecimiento económico, con equidad y sustentabilidad ambiental, y planes de contingencia frente a situaciones extremas.

Proyectos, servicios a terceros y prácticas de extensión

EI PAS trabaja desde hace varios años articuladamente con la SESyC la evaluación de la pertinencia de los proyectos de extensión que lo seleccionan como programa vinculado en sus presentaciones a las diferentes convocatorias. Dada la dimensión multidisciplinaria de la temática ambiental, el número de proyectos seleccionados por el PAS crece en cada convocatoria. Además, los distintos miembros del PAS han presentado proyectos de extensión a diferentes convocatorias. Actualmente se desarrollan cuatro Proyectos de Extensión de Interés Social (PEIS) en diferentes localidades de nuestra región relacionados con humedales naturales y desarrollo local: La Guardia, Alto Verde, La Boca (Santa Fe).

Asimismo, durante 2021 se ha aprobado el Proyecto de Extensión de Interés Institucional (PEII) "Paisaje de Islas: el patrimonio y el turismo como estrategia de desarrollo solidario", que busca contribuir a la puesta en valor del patrimonio de nuestras islas: en particular del Paraje La Boca (ciudad de Santa Fe) en el valle de inundación del Paraná como recurso para el desarrollo sustentable y solidario. La Boca es un barrio del distrito la Costa con capacidad de transformarse en caso demostrativo de una estrategia a ampliar a otros barrios de Santa Fe: Alto Verde, la Vuelta del Paraguayo, la Guardia, Colastiné Sur y Norte, y en ciudades vecinas como San José del Rincón, Arroyo Leyes o Monte Vera, con la que se comparte el área insular del valle de inundación del río Paraná, denominada Microrregión Insular (MRI). La UNL pretende dar continuidad al diálogo de saberes y un nuevo impulso a las acciones de articulación de equipos interdisciplinarios con la comunidad en el marco de la Red Social e Interinstitucional de Alto Verde, acciones que se vienen desarrollando respecto de las temáticas que se plantea abordar en este proyecto. El paisaje insular, patrimonio de patrimonios, es un concepto holístico y transversal con énfasis en la dimensión cultural del desarrollo sustentable que, como idea fuerza territorial, tiene la capacidad de: a) promover el fortalecimiento de la propia identidad y ser oportunidad para reconocer patrimonios valiosos; b) ser vector para el desarrollo local y oportunidad para microeconomías solidarias, turismo comunitario, movilidad fluvial y actividades sustentables; y c) ser instrumento para la gobernanza y oportunidad de acuerdos en la gestión territorial. A través de talleres de capacitación, intervención en espacio público en tierra y agua, relevamientos e inventarios, elaboración de materiales didácticos y de difusión, y diseño de productos turísticos de base comunitaria, se llevará adelante un programa de trabajo resultante de la concertación de intereses con los actores sociales participantes, instituciones y vecinos del paraje, con el aval municipal y provincial en una metodología de participación colectiva y la construcción de consensos sobre una visión compartida del territorio a partir de una idea fuerza territorial: el paisaje de islas.

Otra estrategia del PAS es la realización de Servicios a Terceros, con grupos de docentes de diferentes disciplinas de la Universidad y mediante la integración de unidades académicas en un trabajo en conjunto, como fue el caso del "Relevamiento biológico preliminar de la Reserva Natural Urbana del Oeste, ciudad de Santa Fe, Argentina: unidades de vegetación, flora vascular, fauna de vertebrados (excepto aves) y propuesta de restauración ambiental", que se realizó para la Municipalidad de Santa Fe en el marco de un Financiamiento del Fondo Francés para el Medio Ambiente. 


\section{Docencia y PAS}

A lo largo de estos 15 años el PAS ha generado una nutrida oferta de grado, posgrado y cursos abiertos a la comunidad, como han sido y son los casos de las asignaturas Educación Ambiental, Tecnología Ambiente y Sociedad, Análisis de la Sustentabilidad Ambiental de Proyectos (con enfoque aprendizaje-servicio), Problemáticas Socioambientales Contemporáneas, y los cursos de posgrado Agua y Sociedad y Gestión Ambiental de Proyectos.

En años recientes, el PAS ha elaborado propuestas interdisciplinarias orientadas a la comunidad, como los Cursos de Turismo Consciente y Gestión Ambiental del Territorio. Este último busca brindar criterios y conceptos básicos relacionados con la gestión ambiental territorial mediante diferentes herramientas e instrumentos a tener en cuenta a la hora de llevar adelante un proceso de control y gestión ambiental. La propuesta sobre Turismo Consciente lo aborda como motor de desarrollo sustentable y explora las posibilidades del patrimonio natural y cultural como recurso especialmente en nuestra región.

El desarrollo del ciclo de encuentros 2020 "La fuerza de los paisajes cotidianos. Miradas latinoamericanas en red", en el eje Ambiente y Desarrollo de América Latina de la Cátedra Abierta de Estudios Latinoamericanos "José Martí" (CAELJM) de la Secretaría de Desarrollo Institucional e Internacionalización de la UNL, se propuso un enfoque territorial ambiental con la multidimensionalidad del desarrollo sustentable y el énfasis en las particularidades que aporta la cultura. El concepto de paisaje, considerado patrimonio de patrimonios, constituyó la herramienta teórica y metodológica para indagar la vida cotidiana en casos mexicanos, colombianos y argentinos en exposiciones y debates. ${ }^{4}$

\section{PAS y comunicación}

Este programa, en el trabajo conjunto con la SESyC, hace particular hincapié en el papel de la comunicación en la cuestión ambiental. La comunicación ambiental en todas sus formas complementa a la educación en tanto colabora en la formación de ciudadanos conscientes de las crisis ambientales por las que atravesamos y capaces de minimizar su impacto con la modificación de conductas individuales que se transforman en cambios colectivos. Campañas, diseño de páginas web, actividad en redes sociales, visibilización del calendario ambiental, podcast, micros radiales, espacios en los medios de comunicación audiovisuales de la Universidad, diseño de folletos y publicaciones han sido y son elementos fundamentales para el PAS. Página web de Reservas ${ }^{5}$ y Microrregión Insular ${ }^{6}$ y Colección de Postales del Patrimonio Natural y Cultural de la Microrregión Insular.

\section{El caso de la MRI como laboratorio a cielo abierto}

El caso seleccionado para desarrollar el proceso de investigación es un ambiente insular (Figura 3) subdesarrollado, con un estilo de desarrollo insustentable, que integra la Región Centro en Argentina. Localizado en la Región Metropolitana Santa Fe-Paraná, se encuentra delimitado por el canal de acceso al Puerto de Santa Fe, el río Paraná, el Arroyo Leyes, la 
Laguna Setúbal y el Canal Derivación Norte. Su jurisdicción es compartida por la Nación, la Provincia de Santa Fe, las Municipalidades de Santa Fe y San José del Rincón y la Comuna de Arroyo Leyes. En la actualidad tiene una superficie de $635 \mathrm{~km}^{2}$, una población de alrededor de 60000 habitantes y el proceso de urbanización reproduce los patrones de desarrollo de la ciudad de Santa Fe.

El proyecto parte de que la opción es contribuir a construir una nueva sociedad sustentable (Herrera et al., 2004) que internalice el paradigma ambiental y actúe en consecuencia. Se supone que la construcción de una "mejor sociedad" requiere entre otras acciones de las cinco estrategias siguientes (Mihura, 2020), cuyo diseño y elaboración constituye uno de los productos relevantes del proceso de investigación: Estrategia 1 (E 1) Considerar al ambiente cómo unidad de desarrollo (Mihura, 2010); E 2 Imaginar y proyectar sociedades que nos permitan lograr y mantener armonía con los sistemas no construidos por nuestra especie (Pesci, et al.; 2007); E 3 Analizar conflictos y riesgos ambientales, E 4 Materializar procesos de desarrollo sustentable para construir sociedades de flujos cíclicos (Max-Neff et al.,1993; Pesci et al.; 2007; Mihura, 1993); E 5 Cambiar paradigmas de formación académica, ciencia y tecnología (Pesci et al.; 2007; Checkland; 1993).

Figura 3. Microrregión Insular Santa Fe, San José del Rincón y Arroyo Leyes.

Microunidad de análisis y desarrollo.

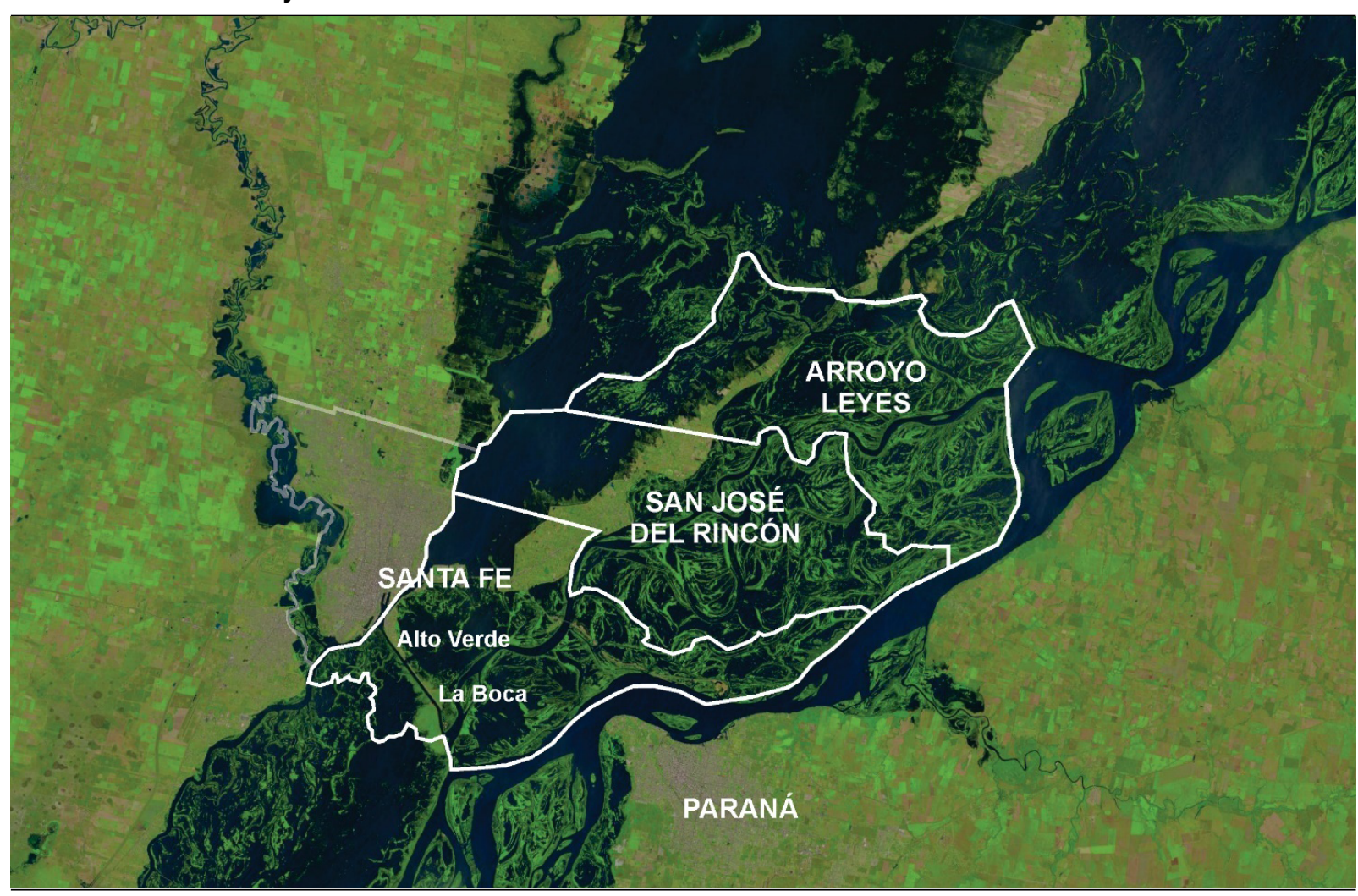

Fuente: PAS. 
Entre las dificultades encontradas, la jurisdicción compartida del área de estudio por tres administraciones locales, la Provincia y la Nación, implica la fragmentación de funciones y visiones sesgadas de los actores; la ausencia de planificación de mediano y largo plazo y/o su no implementación por parte de cada gobierno; los cambios de autoridades en ausencia de políticas de Estado; la resistencia social al cambio producto de la falta de confiabilidad en la dirigencia política y sectorial; la diversidad y disparidad de interlocutores; la fragmentación sectorial; una cultura marcadamente individualista; la lenta implementación del tema-acción (tema generador) "Itinerarios y Travesías" (circuito microrregional) identificado, que posee la capacidad de generar patrones de cooperación entre organizaciones institucionales de gobierno, de formación académica, ciencia y tecnología y de capital, de las cinco jurisdicciones; las limitaciones al funcionamiento transdisciplinario y al enfoque técnico político, soportes de los procesos de investigación interactiva, que son necesarios para la resolución de los conflictos complejos, lo cual impone el paradigma de ciencia y tecnología vigente en las universidades públicas como la nuestra.

Los avances alcanzados con relación al desarrollo de "procedimientos de gestión del desarrollo sustentable en áreas inundables con gobernabilidad difusa", en particular en lo inherente a políticas públicas, organización institucional, planes y acciones prioritarias, se traducen en productos alcanzados en el desarrollo del proceso de investigación interactiva en el caso seleccionado: equipo/espacio central de investigación interactiva conformado; definición técnico-política de la unidad de análisis y desarrollo (caso de la Microrregión Insular de Santa Fe, San José del Rincón y Arroyo Leyes); relevamiento y desarrollo de cartografía: planos urbanos de las localidades de Santa Fe, San José del Rincón y Arroyo Leyes, de áreas con riesgo hídrico (Ley Provincial 11.730), de zonificaciones de la Ley Nacional de Bosques 26.331, de infraestructuras de defensas contra inundaciones, de curvas de nivel territorial y de máximas crecidas registradas; acuerdo sociopolítico sobre los ejes del desarrollo sustentable de la MRI: reconocimiento, cuidado y manejo del patrimonio natural y cultural, definición de modos de habitar, uso del suelo y actividades sostenibles, acuerdos de gobernabilidad y políticas; acta constitutiva de la MRI y de su mesa técnico-política a partir de la realización de reuniones de la Universidad con los gobiernos, provincial y locales. Inclusión de la MRI en la Mesa del Corredor Turístico de la Costa Santafesina del Plan Provincial de Turismo de Santa Fe por parte de la Secretaría de Turismo del Ministerio de la Producción del Gobierno de Santa Fe; acuerdo sociopolítico sobre la problemática principal: incremento del riesgo de inundación como consecuencia de aumento de la vulnerabilidad del área y debido a procesos de desarrollo socioeconómico insustentables; acuerdos académicos alcanzados para avanzar en la conformación de un laboratorio a cielo abierto para experiencias de desarrollo sustentable. Asimismo, se está avanzando en la propuesta de turismo comunitario en el barrio Alto Verde y el Paraje La Boca conjuntamente con el Programa de Economía Social y Solidaria de la SESyC. ${ }^{7}$

Siguiendo esta línea de articulación interdisciplinaria, se han asociado a este proceso los otros equipos de investigación del Proyecto CAl+D Orientado 2016 "Conectando Santa Fe: Movilidad fluvial como oportunidad de integración urbana. El caso de barrio Alto Verde en el 
marco de la Microrregión Insular (MRI)", de la Facultad de Arquitectura, Diseño y Urbanismo (FADU-UNL) (director Bruno Reinheimer) y CAI+D 2020 "Infraestructuras de accesibilidad insular en Santa Fe. Criterios proyectuales para un habitar sustentable en el valle de inundación del Paraná" (directora Patricia Mines).

En el caso de la Microrregión Insular de Santa Fe, San José del Rincón y Arroyo Leyes, se ha logrado mediante diferentes dispositivos de articulación y actividades integradoras generar, sostener y profundizar la asociación operativa entre la UNL y los gobiernos provincial de Santa Fe, municipales de Santa Fe y San José del Rincón, y comunal de Arroyo Leyes, y desde allí se viene trabajando con una estrategia principal (no excluyente) de turismo sustentable que ha permitido interactuar en diferentes líneas de acción con establecimientos educativos, comisiones vecinales, micro y pequeñas empresas y la ciudadanía en general del área.

La factibilidad de poner en marcha procesos de desarrollo sustentable se encuentra asociada a la existencia de decisiones políticas instrumentadas bajo la coordinación de equipos de gestión y planificación político-técnicos, con capacidad de traducir el concepto de ambiente en un sistema de referencias que permita su intervención. Así también de un sistema de gobierno (gobernanza) que implemente estrategias de gestión múltiples y simultáneas en distintos niveles de organización del sistema ambiental abordado. En este sentido, se trabaja actualmente en un acuerdo (convenio en trámite) con el Ministerio de Ambiente y Cambio Climático del Gobierno de Santa Fe para la elaboración conjunta de un plan de desarrollo sustentable y aumento de la resiliencia para la MRI.

\section{Consideraciones finales}

Los 15 años del PAS se desarrollan entre problemáticas ambientales ya tradicionales y otras emergentes de la actualidad. Las actividades de extensión, docencia e investigación, llevadas a cabo han permitido al programa crecer y transformarse en un espacio de transdisciplina de referencia para la comunidad del área de influencia de la Universidad. Enfocado en lograr un desarrollo de la población que integre armónicamente la cultura y la naturaleza, el PAS es consciente de la complejidad de los problemas actuales y teje continuamente redes para integrar a todos los actores posibles para soluciones reales. Una característica distintiva es que se ha trabajado en todos los niveles factibles: con el poblador local en su singularidad, con las comunidades en sus diferentes dimensiones, con las instituciones educativas, con las organizaciones de la sociedad civil y con los distintos gobiernos en la búsqueda de un mejor futuro ambiental. Las limitaciones, fracasos y éxitos de este programa son insumos para seguir adelante y para reconstruir de modo permanente las relaciones del ambiente y la sociedad.

\section{Referencias bibliográficas}

Bordieu, P. (2011). Las estrategias de la reproducción social. Siglo XXI Editores.

Bru, J. (1997). Medio ambiente, poder y espectáculo: gestión ambiental y vida cotidiana. Icaria.

Checkland P. (1993). Pensamiento de Sistemas, Práctica de Sistemas. Limusa. 
Comisión Mundial del Medio Ambiente y el Desarrollo/Organización de las Naciones Unidas (ONU) (1987). Nuestro Futuro Común. Alianza.

Crutzen, P. J. \& Stoerme, E. F. (2000). The "Anthropocene". Global Change Newsletter, (41), 17-18,

Folch, R. y Bru, J. (2017). Ambiente, territorio y paisaje. Valores y valoraciones. Barcino.

Gallopín, G. (2003). Sostenibilidad y desarrollo sostenible: un enfoque sistémico. CEPAL Serie Medio Ambiente y Desarrollo, 64. ONU.

Gallopín, G. (2006). Los indicadores de desarrollo sostenible: aspectos conceptuales y metodológicos. Seminario de expertos sobre indicadores de sostenibilidad en la formulación y seguimiento de políticas. FODEPAL.

Herrera, A. O.; Scolnick, H.; Chichilnisky, G.; Gallopin, G. C.; Hardoy, J.; Mosovich, D.; Oteiza, E.; De Romero Brest, G.; Suárez, C. E. y Talavera, L. (2004). ¿Catástrofe o Nueva Sociedad? Modelo Mundial Latinoamericano. Centro Internacional de Investigaciones para el Desarrollo.

Max-Neef, M.; Elizalde, M. y Hopenhayn, M. (1993). Desarrollo a Escala Humana. Nordan, Comunidad.

Menendez, G. (2013). La dimensión comunicacional de la extensión universitaria. En Menendez, G. et al. Integración docencia y extensión. Otra forma de aprender y enseñar. Ediciones UNL.

Menendez, G. y Tarabella, L. (2017). El aprendizaje experiencial: una práctica de innovación que se afianza en la UNL. En Menendez, G. et al. Integración, Docencia-Extensión 2: Otra forma de enseñar y aprender. Ediciones UNL.

Mihura E. R. (2020). Procedimientos de Gestión del Desarrollo Sustentable en Áreas Inundables con Gobernabilidad Difusa. Informe Final del CAI+D 2016. FICH-UNL.

- (2010). Reflexiones y aportes para la sustentabilidad de procesos de gestión alternativa de la Educación Superior: estrategias para una Educación para el Desarrollo Sustentable (DS). Tesis de Maestría en DS. FLACAM-UNLa.

_ (1993). Proyecto Final. Curso de Posgrado en Formación Ambiental. Facultad Latinoamericana de Ciencias Ambientales. Auspicio UNESCO.

Molina Neira, B. (2018). La incorporación de la cultura y el patrimonio en el desarrollo sostenible desafíos y posibilidades. Humanidades: Revista de la Escuela de Estudios Generales, 8(1).

ONU (Organización de las Naciones Unidas) (2010). El aporte de las Naciones Unidas a la globalización de la ética. Revisión de algunas oportunidades. Unidad Conjunta CEPAL/OACDH.

(2018). La Agenda 2030 y los Objetivos de Desarrollo Sostenible: una oportunidad para América Latina y el Caribe (LC/G.2681-P/Rev.3). https://www.un.org/sustainabledevelopment/es/sustainable-development-goals/

Pesci, R. O. (2010). Los Caminos de la Sustentabilidad. Conferencia Central. 1er. Foro Nacional Argentino del Foro Latinoamericano de Ciencias Ambientales, Cátedra UNESCO para el Desarrollo Sustentable. FICH-UNL. Pesci, R. O.; Perez, J y Pesci, L. (2007). Proyectar la sustentabilidad: Enfoque y metodología de FLACAM. Cepa.

Sabaté Bel, J. (2004). Paisajes culturales. El patrimonio como recurso básico para un nuevo modelo de desarrollo. URBAN 9. Revista del Departamento de Urbanismo y Ordenación del Territorio ETSAM UPC. Universidad Politécnica de Cataluña.

UNL (Universidad Nacional del Litoral) (2013). Estatuto UNL. 